\title{
DETERMINATION OF TEMPERATURE IMPACT ON CMM RESIDUAL KINEMATIC ERRORS DISTRIBUTION
}

\author{
Wiktor Harmatys ${ }^{1}$, Piotr Gąska ${ }^{1}$, Adam Gąska' ${ }^{1}$, Maciej Gruza' ${ }^{1}$, Jerzy Sładek${ }^{1}$ \\ 1 Laboratory for Coordinate Metrology (M-10), Faculty of Mechanical Engineering, Cracow University of \\ Technology, Jana Pawła II 37, 31-864 Kraków, Poland, e-mail: wiktorharmatys@gmail.com; pjgaska@gmail. \\ com; agaska@mech.pk.edu.pl; gruzam@interia.pl; sladek@mech.pk.edu.pl
}

Received: 2015.04.10

Accepted: 2015.05.08

Published: 2015.06.01

\begin{abstract}
In case of coordinate machines that use CAA correction matrix, the issue of kinematic errors analysis may be based on the determination of residual error distribution. Temperature changes have an impact on CMM kinematic structure, which may cause the differences in the map of residual errors. As for today, the residual errors were analysed only for the reference temperature. No research was undertaken on the residual errors changes depending on the temperature variations. This paper presents the experiment aimed at residual errors analysis and resulting errors distributions for different temperatures.
\end{abstract}

Keywords: residual errors, CMM correction.

\section{INTRODUCTION}

Today, in the era of continuous development of technology and industry, it becomes even more important to ensure adequate quality of manufactured products. This task is entrusted to Coordinate Metrology which is an essential tool for quality control in modern companies. The biggest advantage of Coordinate Measuring Technique (CMT) is probably its versatility. Since information about the measured object geometrical features are obtained by determining coordinates of points on the object's surface, one device can be used for many measurement tasks. The main tool of CMT is Coordinate Measuring Machine (CMM). In the CMMs the kinematic pairs can shift in mutually perpendicular directions, which determine the Cartesian axes $x, y, z$ and defining the machine basic coordinate system. Thanks to utilization of different types of probing systems, measurements with CMMs may be automated. The unquestionable advantage of CMMs is their high accuracy and repeatability. The most precise machines offer accuracy up to tenths of a micron, and the works on machines that would be capable of measuring in nanometres are in progress.
However, the problem of CMMs accuracy is still important, especially evaluation of measurement uncertainty. Existing methods of uncertainty estimation recommended for CMMs are costly, especially due to necessity of multiple repetitions of measurements and the cost of required standards. Moreover, these methods require the personnel with certain knowledge and skills of percipient analysis of the results. Simulation methods of uncertainty estimation are different approach to the problem. Their functioning is most often connected with the development of the so-called virtual machines, which are responsible for multiple simulation of measuring task. Simulation methods have proved to be an interesting solution, but they are used almost exclusively in the laboratories of the best metrological research centres [1,2]. This fact is caused by a number of factors, however, the most important ones are: duration of the tests which proceed the preparation of virtual machine for a specific CMM model, and the variability of the conditions in which the machine is working. The models developed so far assume machine functioning at reference conditions (temperature of $20^{\circ} \mathrm{C}$ ). Deviations from this temperature may cause changes in the geometric parameters, espe- 
cially those expressing the length which in turn leads to the variability of the CMM kinematic errors. Currently, at the Laboratory of Coordinate Metrology (LCM) the research are undertaken on the virtual machine which would take into consideration both of mentioned arguments. Developed at LCM virtual machine consists of two main modules: one responsible for machines kinematics modelling and one devoted to probing systems functioning. Distribution of residual errors is a basis for module responsible for kinematic errors modelling, it can be also used in determination of the optimal position of measured object in the machine measuring volume [3]. The paper describes the experiment that involved determination of residual errors distribution in different temperatures, in order to check the influence of temperature variability on kinematic errors. The obtained results as well as conclusions are also presented in the paper.

\section{THE ERRORS RELATED TO CMM KINEMATICS}

Machine's kinematic errors are one of the main factors affecting the accuracy of any CMM. CMM kinematic errors were the subject of a number of studies $[4,5]$ which resulted in the development of models of machine errors, for example full rigid body or reduced rigid body models. The most commonly used description of CMM geometrical errors include 21 components:

- positioning errors for each machine axis $-\mathrm{xtx}$, yty, ztz;

- errors of mutual perpendicularity - xwy, xwz, ywz;

- translation errors - xty, xtz, ytx, ytz, ztx, zty;

- rotation errors - xry, xrz, yrx, yrz, zrx, zry, xrx, yry, zrz.
The geometrical errors defined in such a manner are a basis for operation of CAA (Computed Aided Accuracy) Matrix. The matrix itself as well as the methods of its determination are further described in [6]. The usage of this matrix has become a common practice, because it allows to correct most of the errors components associated with the machine kinematic. The Figure 1 shows the values of individual components before (red bars) and after (green bars) the CAA matrix was used.

As it can be seen, some errors were almost completely corrected. The remaining errors include both random errors and systematic errors that cannot be corrected or their correction would be unviable, namely residual errors. Since most of the currently used machines, utilize CAA correction system, the issue of CMM kinematic error analysis can be transferred to the analysis of residual errors. However, because the residual errors take into account the random errors components, they should be expressed rather by the probability distribution than certain values of error.

The residual errors distribution can be expressed by assigning the standard deviations of points coordinate reproduction to chosen reference points in measuring volume of machine. The standard deviations represent the probability of reference point reproduction. The exemplary distribution of residual points is presented in Figure 2 . The red arrows represent the standard deviation in scale 100000:1. All values are given in [mm].

\section{EXPERIMENT AND RESULTS}

The distribution of residual errors is a basis for operation of one of the modules of virtual machine which is responsible for the kinematic errors modelling. Special methodology for residual errors determination was prepared at LCM for

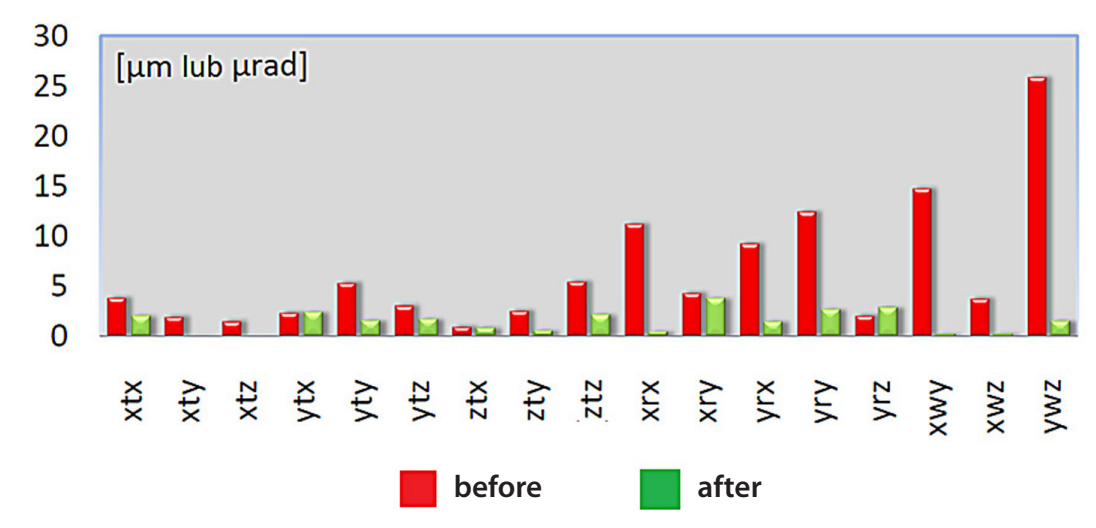

Fig. 1. The geometrical errors before and after applying the CAA correction 


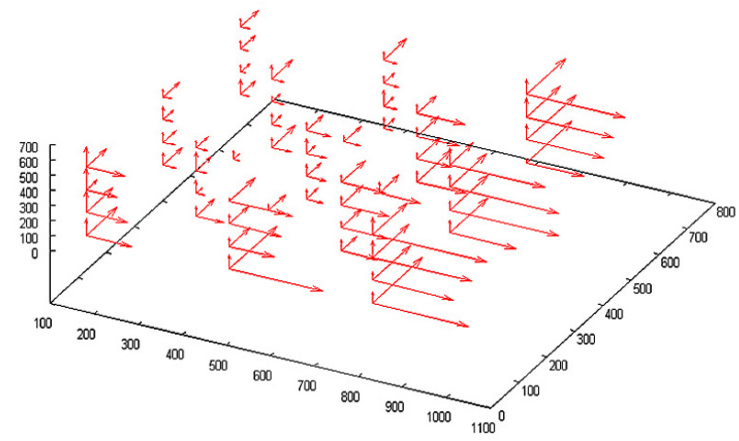

Fig. 2. The example of distribution of residual errors distribution

the purpose of the developed virtual machine [7]. Residual errors are determined in chosen points which defines the reference grid. The required measurements are taken using LaserTracer system which uncertainty for spatial measurements can be expressed by the following equation: $U$ $=0,2 \pm 0,3 \cdot L / 1000 \mu \mathrm{m}$. LaserTracer is a part of laser tracking devices family. It measures the distance to the target (retroreflctor) whose movements it follows. During measurements retroreflector is mounted on the machine in the place of probe head. LaserTracer as a more accurate device is used in order to check if machine indicate a correct coordinates of a reference point. The machine is programmed in such a way that retroreflector approach to each node of reference grid from several directions (14 approach direction for each point) and then moves to the another grid node. As the LaserTracer is able to measure only length, the multilateration method need to be used to determine the coordinate values. That means that the whole measuring sequence should be repeated at least four times, each time placing the Laser Tracer in different position in CMM measuring volume. During all required measurements the CAA matrix installed on the machine have to be active. The described methodology was used to determine the residual errors distribution in different temperatures.

Measurements described in this paper were performed on the Zeiss WMM850S machine, located in the LCM at the Cracow University of Technology. This machine has a moving bridge, and it is characterized by measuring volume of $800 \backslash 1200 \backslash 700 \mathrm{~mm}$. It is placed in the air-conditioned room, which allows temperature control. The three following temperatures for residual errors determination were chosen: $20 \pm 0,2{ }^{\circ} \mathrm{C}$; $21 \pm 0,2{ }^{\circ} \mathrm{C}$ and $22 \pm 0,2{ }^{\circ} \mathrm{C}$. The ambient conditions during all measurements were monitored using temperature sensors which were mounted in measuring volume of the machine and close to the scales corresponding to each axis. The readings from sensors indicate that the temperature during the test was within the specified range. The residual errors were determined in 36 reference points distributed evenly in measuring volume of the machine. The grid nodes nominal coordinates are presented in Table 1.

Table 1. The grid of reference points

\begin{tabular}{|c|c|c|c|c|c|c|c|}
\hline Point number & $\mathrm{x}[\mathrm{mm}]$ & $\mathrm{y}[\mathrm{mm}]$ & $\mathrm{z}[\mathrm{mm}]$ & Point number & $\mathrm{x}[\mathrm{mm}]$ & $\mathrm{y}[\mathrm{mm}]$ & $\mathrm{z}[\mathrm{mm}]$ \\
\hline 1 & 180 & 242 & 64 & 19 & 180 & 242 & 338 \\
\hline 2 & 180 & 627 & 64 & 20 & 180 & 627 & 338 \\
\hline 3 & 180 & 1012 & 64 & 21 & 180 & 1012 & 338 \\
\hline 4 & 440 & 242 & 64 & 22 & 440 & 242 & 338 \\
\hline 5 & 440 & 627 & 64 & 23 & 440 & 627 & 338 \\
\hline 6 & 440 & 1012 & 64 & 24 & 440 & 1012 & 338 \\
\hline 7 & 700 & 242 & 64 & 25 & 700 & 242 & 338 \\
\hline 8 & 700 & 627 & 64 & 26 & 700 & 627 & 338 \\
\hline 9 & 700 & 1012 & 64 & 27 & 700 & 1012 & 338 \\
\hline 10 & 180 & 242 & 201 & 28 & 180 & 242 & 475 \\
\hline 11 & 180 & 627 & 201 & 29 & 180 & 627 & 475 \\
\hline 12 & 180 & 1012 & 201 & 30 & 180 & 1012 & 475 \\
\hline 13 & 440 & 242 & 201 & 31 & 440 & 242 & 475 \\
\hline 14 & 440 & 627 & 201 & 32 & 440 & 627 & 475 \\
\hline 15 & 440 & 1012 & 201 & 33 & 440 & 1012 & 475 \\
\hline 16 & 700 & 242 & 201 & 34 & 700 & 242 & 475 \\
\hline 17 & 700 & 627 & 201 & 35 & 700 & 627 & 475 \\
\hline 18 & 700 & 1012 & 201 & 36 & 700 & 1012 & 475 \\
\hline
\end{tabular}




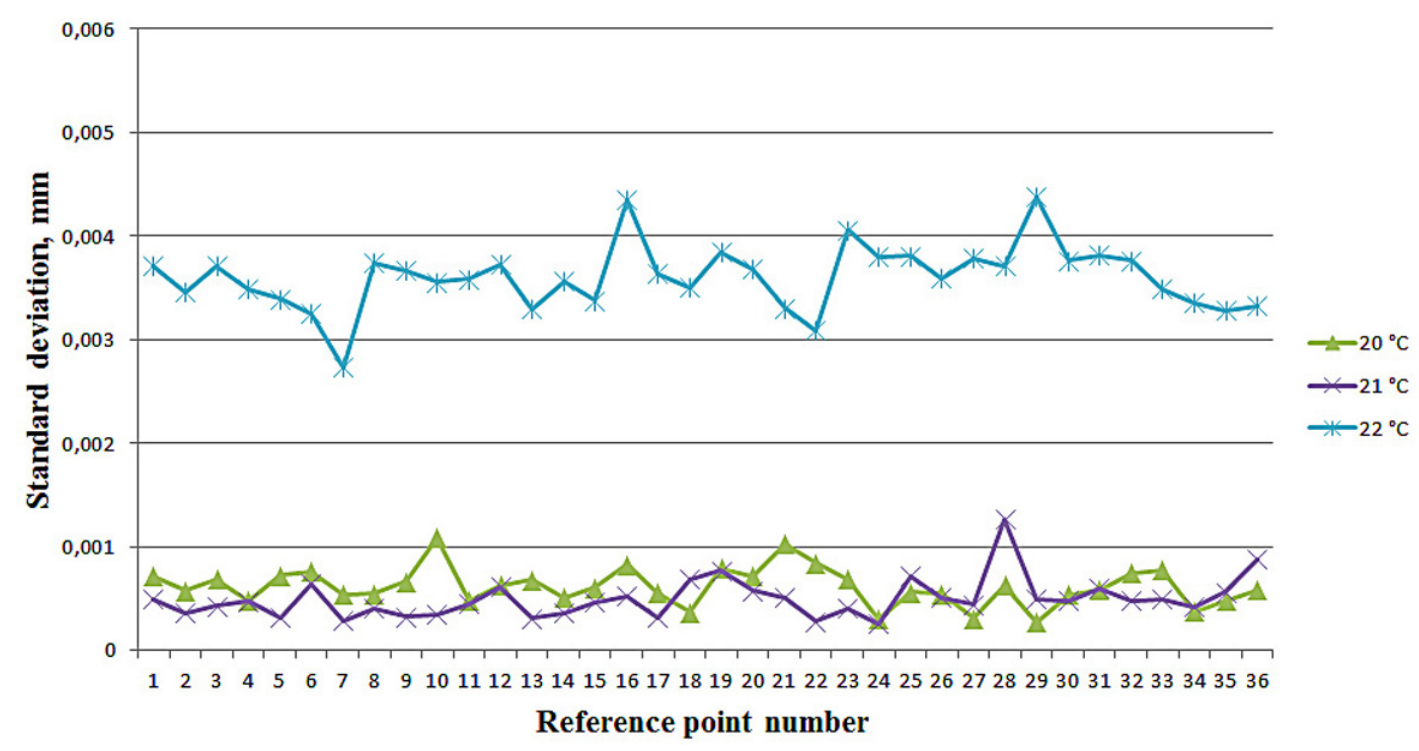

Fig. 3. Standard deviation of point reproduction in $x$-axis, for different temperatures

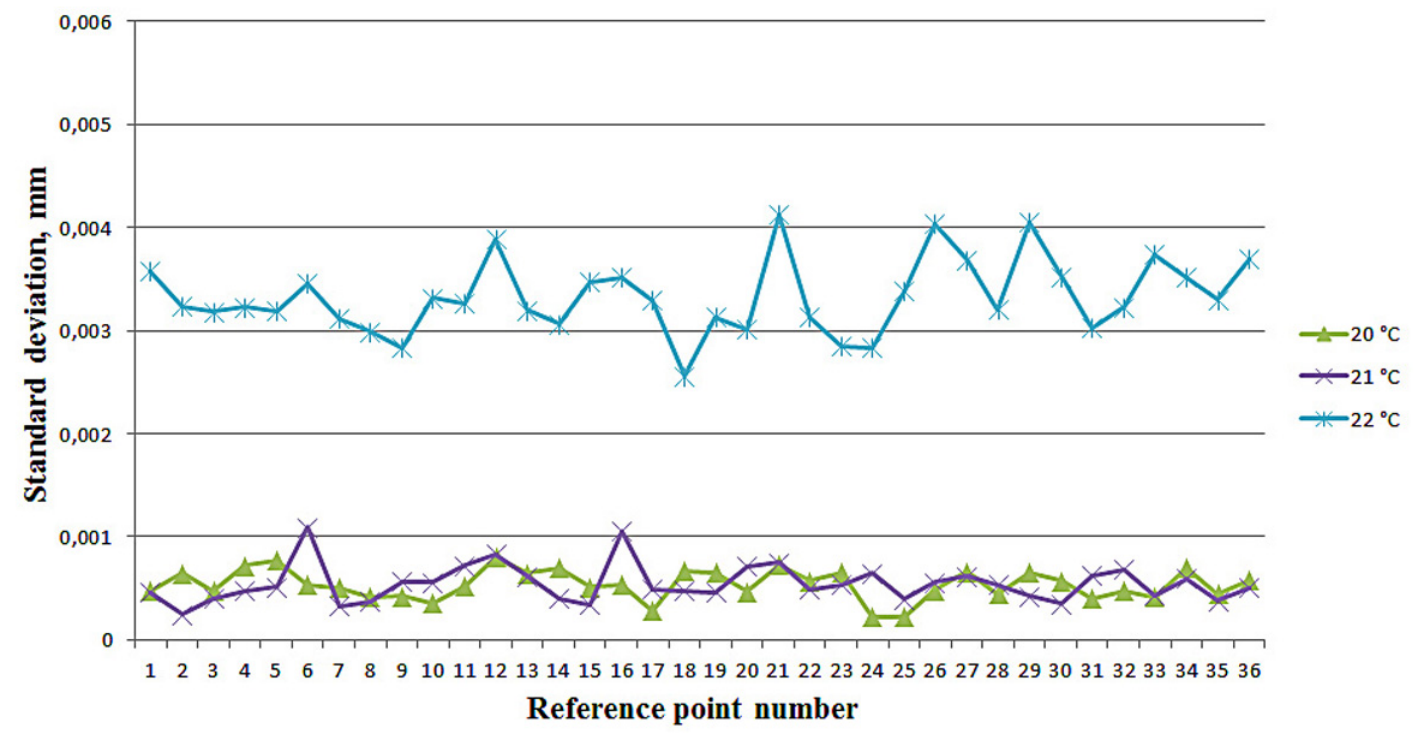

Fig. 4. Standard deviation of point reproduction in $y$-axis, for different temperatures

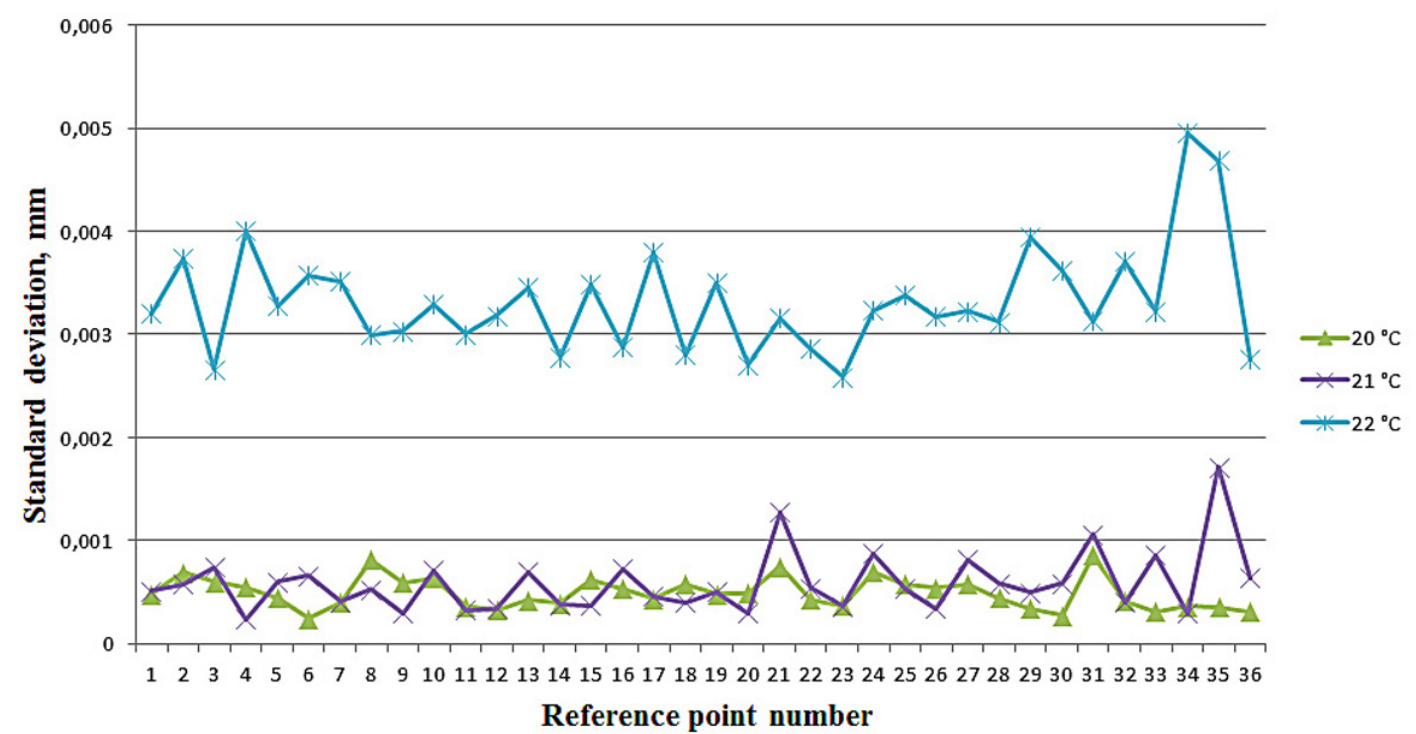

Fig. 5. Standard deviation of point reproduction in $z$-axis, for different temperatures 
The obtained results are presented in Figures $3-5$ which show the standard deviation of each point reproduction respectively in $x, y$ and $z$ axis for all three examined temperatures. Point numbers correspond to the numeration from Table 1.

\section{CONCLUSIONS}

The obtained results indicate that changes in the temperature noticeably affects the residual errors derived from machine kinematics. The results obtained for the $22 \pm 0,2^{\circ} \mathrm{C}$ are characterized by not only the biggest values, but also the highest variability, which reaches two micrometers. Also the results obtained for $21 \pm 0,2{ }^{\circ} \mathrm{C}$, show the considerable volatility. The temperatures bigger than $22^{\circ} \mathrm{C}$ are rarely met in measuring rooms even in industrial conditions so studies at higher temperatures are unnecessary. The further research need to be undertaken in order to verify if the similar results would be obtained also for the temperatures lower than reference temperature of $20^{\circ} \mathrm{C}$, what appears to be likely. If these suppositions are confirmed, it will mean that Virtual machines that could be successfully implemented in the industry should take into consideration thermal influences. In case of virtual machine developed at LCM that means that residual errors distribution (which is a base for a module that simulates the CMM kinematic errors) should be determined for different temperatures. The number of temperatures in which tests ought to be performed should be chosen on the basis of experience of measuring personnel which work with a specified CMM model.

\section{Acknowledgements}

Reported research was realized within confines of project financed by Polish National
Center for Research and Development No: LIDER/06/117/L-3/11/NCBR/2012.

\section{REFERENCES}

1. Trapet E., Franke M., Hartig F., Schwenke H., Wadele F., Cox M., Forbers A., Delbressine F., Schnellkens P., Trenk M., Meyer H., Morltz G., Guth Th., Wanner N.: Traceability of coordinate measuring machines according to the method of the Virtual Measuring Machines, PTB-F-35, Germany, Braunschweig, 1999.

2. Sładek J.: Modelowanie i ocena dokładności maszyn oraz pomiarów współrzędnościowych. Praca habilitacyjna, Wydział Mechaniczny, Politechnika Krakowska, 2001.

3. Gąska A., Krawczyk M., Kupiec R., Ostrowska K., Gąska P., Sładek J.: Modeling of the residual kinematic errors of coordinate measuring machines using LaserTracer system, The International Journal of Advanced Manufacturing Technology, 73, 1-4, 2014, 497-507.

4. Trapet E. et al.: Traceability of coordinate measurements according to the method of the virtual measuring machine. Final report, European Commission SMT- EDG XII/C/5 Project No. Mat. 1 CT94-0076.

5. Schwenke H., Knapp W., Haitjema H., Weckenmann A., Schmitt R., Delbressine F.: Geometric error measurement and compensation of machines - An update. CIRP Annals - Manufacturing Technology, 57, 2008, 660-675.

6. Gąska A., Gruza M., Gąska P., Karpiuk M., Sładek J.: Identification and correction of coordinate measuring machine geometrical errors using LaserTracer systems, Advances in Science and Technology Research Journal, 7, 20, 2013, 17-22.

7. Gąska A.: Modeling the accuracy of coordinate measurement with use of Monte Carlo method. Ph.D. Dissertation, Cracow University of Technology, 2011. 